\title{
Cytogenetic Profile of De Novo B lineage Acute Lymphoblastic Leukemia: Determination of Frequency, Distribution Pattern and Identification of Rare and Novel Chromosomal Aberrations in Indian Patients
}

\author{
Prerana Bhandari ${ }^{1}$, Firoz Ahmad ${ }^{1}$, Rupa Dalvi ${ }^{2}$, Neeraja Koppaka ${ }^{2}$, Prajakta \\ Kokate $^{2}$, Bibhu Ranjan Das ${ }^{1}$, Swarna Mandava ${ }^{2 *}$
}

\begin{abstract}
Background: Chromosomal aberrations identified in acute lymphoblastic leukemia (ALL) have an important role in disease diagnosis, prognosis and management. Information on karyotype and associated clinical parameters are essential to physicians for planning cancer control interventions in different geographical regions. $\underline{\text { Materials }}$ and Methods: In this study, we present the overall frequency and distribution patterns of chromosomal aberrations in both children and adult de novo B lineage ALL Indian patients using conventional cytogenetics, interphase FISH and multiplex RT-PCR. Results: Among the 215 subjects, cytogenetic results were achieved in $172(80 \%)$ patients; normal karyotype represented $37.2 \%$ and abnormal $62.8 \%$ with a distribution as follows: $15.3 \%$ hypodiploidy; $10.3 \%$ hyperdiploidy; $15.8 \% \mathrm{t}(9 ; 22) ; 9.8 \% \mathrm{t}(1 ; 19) ; 3.7 \% \mathrm{t}(12 ; 21) ; 2.8 \% \mathrm{t}(4 ; 11) ; 2.8 \%$ complex karyotypes. Apart from these, we observed several novel, rare and common chromosomal rearrangements. Also, FISH studies using LSI extra-signal dual-color probes revealed additional structural or numerical changes. Conclusions: These results demonstrate cytogenetic heterogeneity of ALL and confirm that the incidence of chromosomal abnormalities varies considerably. To the best of our knowledge, this is one of the largest reported series of cytogenetic investigations in Indian B-lineage ALL cases. In addition, ongoing cytogenetic studies are warranted in larger groups of B-lineage ALL cases to identify newly acquired chromosomal abnormalities that may contribute to disease diagnosis and management.
\end{abstract}

Keywords: Acute lymphoblastic leukemia - cytogenetic analysis - fluorescence in situ hybridization

Asian Pac J Cancer Prev, 16 (16), 7219-7229

\section{Introduction}

Around $80 \%$ of all childhood acute leukemia is ALL, especially B-cell-precursor acute lymphoblastic leukemia (BCP-ALL) (80-85 \% of ALL), which is the leading cause of cancer-related death in children and young adults. In India, this proportion varies between $60-85 \%$ (Arora et al., 2009) and with the current population rates, it is likely to increase. Over the years, there has been a significant improvement in the outcome of ALL patients with a 5-year EFS $>80 \%$ in developed countries (Hunger et al., 2012) and the future is encouraging.

Most ALL patients are genetically heterogeneous, harboring different chromosomal (numerical and/or structural) and/or submicroscopic molecular abnormalities crucial in diagnosis, prognosis, chemo-therapeutic response and outcome (Iacobucci et al., 2012). In turn, their identification contributes towards understanding disease pathogenesis. Their relevance is likely to increase as targeted therapies are introduced, as exemplified by the success of tyrosine kinase inhibitor in the treatment of Philadelphia positive ALL (Pui and Evans, 2006). Current genetic abnormalities of significance in ALL management include: $\mathrm{t}(9 ; 22)(\mathrm{q} 34 ; \mathrm{q} 11)$ (BCR-ABL1), $\mathrm{t}(4 ; 11)(\mathrm{q} 21 ; \mathrm{q} 23)$ (MLL-AFF1 alias MLL-AF4) and near haploid/low hypodiploidy, all of which are poor prognostic markers; t(1;19)(q23;p13) (TCF3-PBX1 alias E2A-PBX1) as an intermediate prognostic marker; while $\mathrm{t}(12 ; 21)$ (p13;q22) (ETV6-RUNX1 alias TEL-AML1) and high hyperdiploidy, are both favorable prognostic markers (Harrison et al., 2010). The presence of recurrent genetic markers represents subtypes of the disease which may have different etiologies.

The frequencies of genetic aberrations differ distinctly in childhood and adult ALL. In childhood ALL, the impact of cytogenetic factors like specific translocations and DNA ploidy is well defined, while the prognostic significance of karyotype in adult ALL is less clear, partially as 
the disease is less frequent. Furthermore, the relative distribution of molecular subtypes in ALL is not uniform in different geographic regions. Thus, we examined the cytogenetic profile of newly diagnosed B-lineage ALL cases to determine the frequency and subtypes of cytogenetic aberrations from an Indian perspective, and their correlation with clinical parameters. An attempt was also made to compare the major aberrations (enlisted by WHO) in childhood and adult Indian ALL patients with Asian and global reports.

\section{Materials and Methods}

\section{Patients}

The present study included 215 de novo B-lineage ALL subjects referred from July 2011-October 2013 and were subjected to cytogenetic and/or molecular analysis of chromosomal rearrangements conducted at SRL Ltd., Mumbai, India. The study was in accordance with the declaration of Helsinki and approved by the Institutional ethics committee. Written consent was obtained from all the subjects.

\section{Cytogenetic analysis}

We karyotyped bone marrow aspirate or whole blood cells withdrawn at diagnosis as per standard unstimulated direct $(0 \mathrm{~h}, 3 \mathrm{~h})$ and short term $(24 \mathrm{~h}, 48 \mathrm{~h})$ cell culture technique previously described (Bhandari et al., 2014). In brief, cells were cultured in RPMI (Sigma, Schnelldorf, Germany) medium supplemented with $20 \%$ FBS (Gibco, Grand Island, NY) at $37^{\circ} \mathrm{C}$. After incubation at respective time intervals, the cells were harvested i.e. cell-growth was arrested by exposure to colchicine (HiMedia, $4 \mathrm{mg}$ / $\mathrm{ml}$ ) for 45 minutes, then hypotonised by $0.075 \mathrm{M} \mathrm{KCl}$ for 20 minutes, then fixed with chilled Carnoy's fixative (methanol: glacial acetic acid 3:1). Fixed cells were dropped on chilled frosted slides, then aged overnight at $60^{\circ} \mathrm{C}$ and stained for GTG banding at resolution of about 400-band level. At least 20 metaphase plates were screened, and 4-5 well spread metaphases were photographed and karyotyped using Ikaros Software (MetaSystems $\mathrm{GmbH}$, Germany). The karyotype was interpreted according to ISCN 2013 (ISCN 2013) guidelines.

Fluorescence In Situ Hybridization (FISH) Analysis

FISH was performed on selected cases to refine complex or ill-defined rearrangements identified by chromosome analysis and to confirm recurrent /cryptic chromosomal aberrations such as BCR-ABL, ETV6RUNX1, and MLL rearrangements, using LSI Vysis Probes (Abbott Molecular, Des Plaines, IL). The slides were hybridized overnight according to Vysis protocol and analyzed using Isis Software (MetaSystems GmbH, Germany).

\section{Molecular Analysis for Detection of Gene Fusion Transcripts}

To confirm /reveal recurrent or cryptic chromosomal rearrangements such as $\mathrm{t}(9 ; 22)(\mathrm{q} 34 ; \mathrm{q} 11), \mathrm{t}(1 ; 19)$ $(\mathrm{q} 23 ; \mathrm{p} 13), \mathrm{t}(4 ; 11)(\mathrm{q} 21 ; \mathrm{q} 23)$ and $\mathrm{t}(12 ; 21)(\mathrm{p} 13 ; \mathrm{q} 22)$ in cases with apparently normal karyotype or with insufficient metaphases, multiplex nested reverse transcriptase PCR with minor modification (Pallisgaard et al., 1998) was performed. Briefly, fifty nanograms of total RNA extracted using QIAamp RNA blood mini kit (Qiagen, Hilden, Germany) used to prepare cDNA using random hexamer primer (Fermentas, Hanover, MD) and Sensiscript RT Kit (Qiagen). This was followed by two rounds of nested RT-PCR for detection of the fusion genes. Appropriate internal control was also analysed to check the integrity of RNA.

\section{Statistical analysis}

The $\chi^{2}$ test and Fisher's exact test were used to compare the categorical data between adult and pediatric ALL patients. Z-test was performed to compare the frequencies of different abnormalities with available published reports of different countries. Two-side P-values $<0.05$ were considered statistically significant.

\section{Results}

Total 215 newly diagnosed B-lineage ALL patients, comprising of 130 males and 85 females (M:F ratio=1.5:1), with age ranging from $<1-75$ years were analysed (Table 1). The study population consisted of 143 children (aged $\leq 14$ years; $M: F$ ratio $=1.6: 1$ ) and 72 adults (aged $\geq 15$ years; $\mathrm{M}: \mathrm{F}$ ratio=1.4:1). Of the 215 subjects, 172 patients $(80 \%)$ showed successful cultures. Total 135 patients $(62.8 \%)$ showed chromosomal aberrations, while 80 (37.2\%) displayed normal karyotype.

Table 1. Clinical Characteristics of Indian B-lineage ALL Patients

\begin{tabular}{lccc}
\hline Demographic Parameters & Total Cases & Pediatric* Cases & Adult ${ }^{\dagger}$ Cases \\
\hline $\begin{array}{l}\text { Total, } \mathrm{n}(\%) \\
\text { Gender }\end{array}$ & $215(100)$ & $143(66.5)$ & $72(33.5)$ \\
Male, $\mathrm{n}(\%)$ & $130(60.5)$ & $87(61)$ & $43(60)$ \\
Female, $\mathrm{n}(\%)$ & $85(39.5)$ & $56(39)$ & $29(40)$ \\
Age (years), Median (range) & $8(0.01-75)$ & $4(0.01-14)$ & $26.5(15-75)$ \\
Hb level (gm/dl) median (range) & $7.5(1.6-15)$ & $7.4(1.6-15)$ & $7.7(2.5-15)$ \\
Platelets count $\left(\times 10^{\%} / \mathrm{L}\right)$ median (range) & $40(0.7-470)$ & $40(3-470)$ & $37.8(0.7-154)$ \\
WBC count $(\mathrm{x} \mathrm{10} / \mathrm{L})$ median (range) & $17.5(0.2-536)$ & $21(0.9-536)$ & $10.8(0.2-451)$ \\
$<50(\mathrm{x} \mathrm{10} / \mathrm{L}), \mathrm{n}(\%)$ & $154(71.6)$ & $102(71.4)$ & $52(72)$ \\
$\geq 50(\mathrm{x} \mathrm{10} / \mathrm{L}), \mathrm{n}(\%)$ & $61(28.4)$ & $41(28.6)$ & $20(28)$ \\
\hline
\end{tabular}

Hb: Hemoglobin; WBC: white blood cell; *age range: <15years; †age range: $\geq 15$ years 


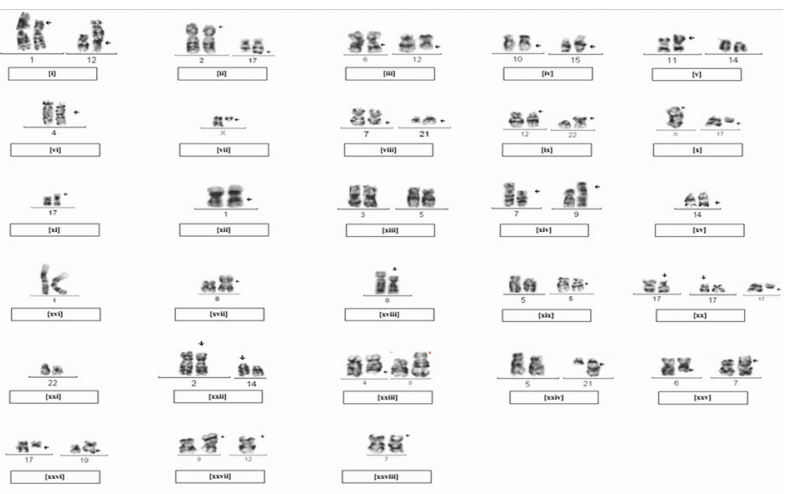

Figure 1. Partial karyotypes of Novel \& Rare Structural Chromosomal Rearrangements Showing only the Chromosomes Involved. [i] $\mathrm{t}(1 ; 12)(\mathrm{p} 34.1 ; \mathrm{q} 24.33)$, [ii] $\mathrm{t}(2 ; 17)(\mathrm{p} 23 ; \mathrm{q} 25)$, [iii] $\mathrm{t}(6 ; 12)(\mathrm{q} 13 ; \mathrm{q} 13)$, [iv] $\mathrm{t}(10 ; 15)(\mathrm{q} 22 ; \mathrm{q} 22)$, [v] $\mathrm{t}(11 ; 14)(\mathrm{p} 15 ; \mathrm{q} 24),[\mathrm{vi}] \operatorname{inv}(4)(\mathrm{p} 14 \mathrm{p} 16)$, [vii] del(X)(q11.1), [viii] $\mathrm{t}(7 ; 21)(? \mathrm{q} 32 ; \mathrm{q} 22.1),[\mathrm{ix}] \mathrm{t}(12 ; 22)(\mathrm{p} 11.2 ; \mathrm{p} 11.2),[\mathrm{x}]$ $\mathrm{t}(\mathrm{X} ;$ ?17)(p22.3;?q11.2), [xi] inv(17)(p11.2p13.1), [xii] i(1)(p10), and [xiii] $\mathrm{t}(3 ; 5)(\mathrm{q} 21 ; \mathrm{q} 31)$, [xiv] $\mathrm{t}(7 ; 9)(\mathrm{p} 14 ; \mathrm{p} 24),[\mathrm{xv}] \operatorname{inv}(14)$ (q11.2q32), [xvi] dup(1)(q21q42), [xvii] i(8)(q10), [xviii] del(8) (p23), [xix] del(5)(p11), del(5)(q33q35), [xx] del(17)(p11.2), $\operatorname{del}(17)(\mathrm{q} 21.1), \operatorname{del}(17)(\mathrm{q} 11.2),[\mathrm{xxi}] \operatorname{del}(22)(\mathrm{q} 12),[\mathrm{xxii}] \mathrm{t}(2 ; 14)$ (?p13-16;q32), [xxiii] t 4 ; 8)(q31;q24.3), [xxiv] t $(5 ; 21)(\mathrm{q} 13 ; \mathrm{q} 22)$, $[x x v] \mathrm{t}(6 ; 7)(\mathrm{q} 21 ; \mathrm{q} 22),[\mathrm{xxvi}] \mathrm{t}(17 ; 19)(\mathrm{q} 21 ; \mathrm{q} 13.3),[\operatorname{xxvii}] \mathrm{t}(9 ; 12)$ (p13;q13), [xxviii] $\operatorname{del}(7)(\mathrm{p} 15.3)$

\section{Distribution and frequencies of chromosomal rearrangements}

Table 2 shows relative frequencies and distribution of B-ALL specific chromosomal rearrangements and their associated clinical characteristics. Amongst 215 patients, the major alterations included hyperdiploidy (10.3\%), hypodiploidy $(15.3 \%), \mathrm{t}(9 ; 22)(15.8 \%), \mathrm{t}(1 ; 19)$ $(9.8 \%), \mathrm{t}(12 ; 21)(3.7 \%), \mathrm{t}(4 ; 11)(2.8 \%)$, other structural changes $(28 \%)$ and complex karyotypes $(2.8 \%)$. Further amongst our pediatric and adult patients, chromosomal abnormalities were more frequent in the adult patients (75\% vs 56.6\%) $(\mathrm{P}<0.05)$.

\section{Numerical chromosomal changes}

Elucidating modal chromosome number at diagnosis is essential to stratify hypo/ hyperdiploid patients into appropriate risk group for treatment. Table 3 shows AgeSex-Modal number distribution observed:- $37.2 \%$ Normal diploid ( $2 n=46$ chromosomes); Hypodiploidy $(2 n<46$ chromosomes) with sub-groups: $1 \%$ Near-haploidy $(2 n=25-29), 6 \%$ Low-hypodiploidy $(2 n=30-39), 8.4 \%$ Moderate-hypodiploidy $(2 \mathrm{n}=40-45)$; and Hyperdiploidy ( $2 n>46$ chromosomes) with sub-groups: $4.2 \%$ Moderatehyperdiploidy $(2 \mathrm{n}=47-50), 3.7 \%$ High-hyperdiploidy $(2 n=51-65), 1 \%$ Near-triploidy $(2 n=66-80)$ and $1.4 \%$ Near-tetraploidy $(2 n=\geq 81)$. Ploidy distribution amongst pediatric and adult groups was mostly alike with few exceptions. Like near-triploidy and near-tetraploidy were seen exclusively only in adults aged $>20$ years. Also, both moderate (5.6\% vs $1.4 \%)$ and high hyperdiploidy (4.9\% vs $1.4 \%$ ) were more frequent in pediatric than adult patients, but these differences were not significant (all $\mathrm{P}>0.1$ ).
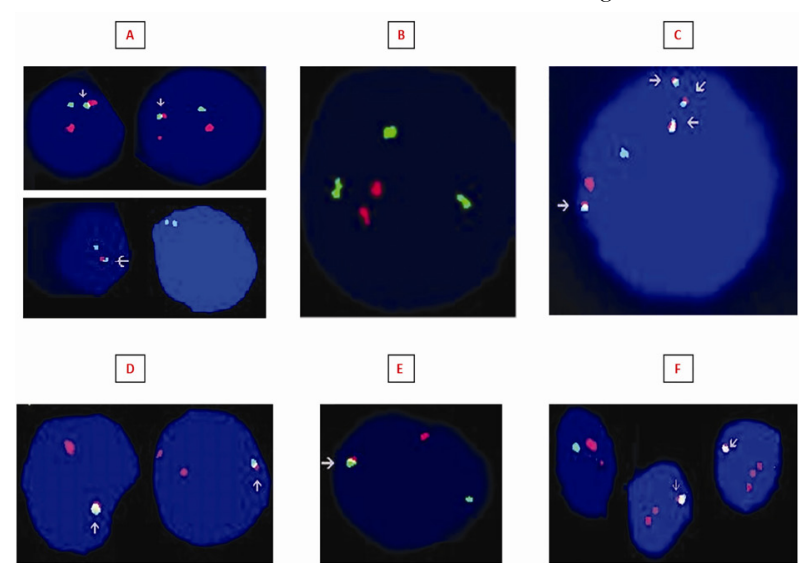

Figure 2. FISH Analysis Using Vysis LSI Probes on Interphase Cells [i] in BCR-ABL positive patients allowed detection of additional alterations like [A] cryptic deletions at ABL breakpoint on chromosome 9(q) (indicated via reduction of 2 red to 1 red signal or by absence of native red signal), [B] multiplication involving the $22 \mathrm{q} 11 \mathrm{BCR}$ locus (indicated by presence of $>2$ green signals) and [C] presence of masked double/ multiple Philadelphia chromosome (indicated by presence of $>1$ fusion signal); and in [ii] cases suspected of ETV6-RUNX1 it allowed detection of [D] deletions of nontranslocated ETV6 allele from 12p13 (indicated via absence of native green signal) [E] deletions of residual RUNX1 allele on chromosome 21 (indicated via reduction of 2 red to 1 red signal), and $[\mathrm{F}]$ multiplication involving the 21q22 RUNX1 locus (indicated by presence of $>2$ red signals)

\section{Structural chromosomal changes}

A higher frequency of structural abnormalities was observed as compared to numerical aberrations in both pediatric (53\% vs $25 \%)$ and adult groups ( $75 \%$ vs $25 \%)$ (all $\mathrm{P}<0.0001$ ). As seen in Table 2 amongst the two groups, adults showed a higher frequency of $\mathrm{t}(9 ; 22)(29.2 \% \mathrm{vs}$ $9.1 \%, \mathrm{P}<0.001)$. In contrast, while $5.6 \%$ pediatric cases showed $\mathrm{t}(12 ; 21)$, it was absent in the adult patients. Slightly elevated $t(4 ; 11)$ was observed in pediatric than adult patients $(3.5 \%$ vs $1.4 \%)$, while almost equal pediatric $(9.1 \%)$ and adult $(11.1 \%)$ cases showed $t(1 ; 19)$ but these differences were not significant (all $\mathrm{P}>0.1$ ).

Partial karyotypes of rare and novel structural chromosomal rearrangements observed are shown in Figure 1. In our study, cytogenetic analysis revealed several novel abnormalities like $\mathrm{t}(1 ; 12)(\mathrm{p} 34.1 ; \mathrm{q} 24.33)$, $\mathrm{t}(2 ; 17)(\mathrm{p} 23 ; \mathrm{q} 25), \mathrm{t}(6 ; 12)(\mathrm{q} 13 ; \mathrm{q} 13), \mathrm{t}(10 ; 15)(\mathrm{q} 22 ; \mathrm{q} 22)$, $\mathrm{t}(11 ; 14)(\mathrm{p} 15 ; \mathrm{q} 24)$, paracentric inv(4)(p14p16) and $\operatorname{del}(\mathrm{X})(\mathrm{q} 11.1)$ in childhood ALL. While we observed $\mathrm{t}(7 ; 21)(? \mathrm{q} 32 ; \mathrm{q} 22.1), \mathrm{t}(12 ; 22)(\mathrm{p} 11.2 ; \mathrm{p} 11.2), \mathrm{t}(\mathrm{X} ; ? 17)$ (p22.3;?q11.2), paracentric inv(17)(p11.2p13.1) and i(1) (p10) among adults. In addition, we observed several rare structural chromosomal rearrangements that were differently distributed among the pediatric and adult patients like $\mathrm{t}(3 ; 5)(\mathrm{q} 21 ; \mathrm{q} 31), \mathrm{t}(7 ; 9)(\mathrm{p} 14 ; \mathrm{p} 24)$, pericentric $\operatorname{inv}(14)(q 11.2 q 32), \operatorname{dup}(1)(q 21 q 42), i(8)(q 10), \operatorname{del}(8)$ (p23), del(5)(p11), del(5)(q33q35), del(17)(p11.2), del(17) (q21.1) and $\operatorname{del}(22)(\mathrm{q} 12)$ were exclusively observed in childhood cases. While $\mathrm{t}(2 ; 14)(? \mathrm{p} 13-16 ; \mathrm{q} 32), \mathrm{t}(4 ; 8)$ $(\mathrm{q} 31 ; \mathrm{q} 24.3), \mathrm{t}(5 ; 21)(\mathrm{q} 13 ; \mathrm{q} 22), \mathrm{t}(6 ; 7)(\mathrm{q} 21 ; \mathrm{q} 22), \mathrm{t}(9 ; 12)$ $(\mathrm{p} 13 ; \mathrm{q} 13), \mathrm{t}(17 ; 19)(\mathrm{q} 21 ; \mathrm{q} 13.3), \operatorname{del}(7)(\mathrm{p} 15.3)$ and del(17q11.2) were observed only in adult patients. 


\begin{tabular}{|c|c|c|c|c|c|c|c|c|c|c|c|c|c|}
\hline \multirow{6}{*}{$\begin{array}{l}\tilde{d} \\
\tilde{w} \\
0\end{array}$} & 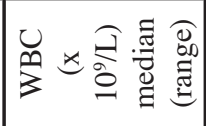 & 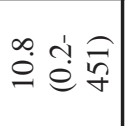 & 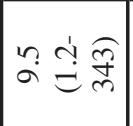 & 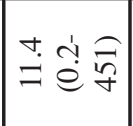 & 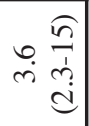 & $\stackrel{m}{i}$ & 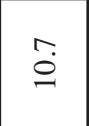 & $\stackrel{n}{\stackrel{9}{1}}$ & i․ & 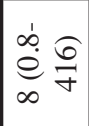 & 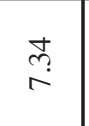 & 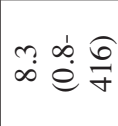 & 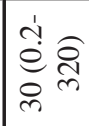 \\
\hline & 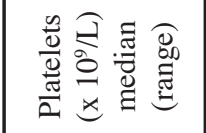 & 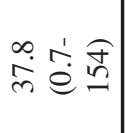 & 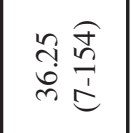 & 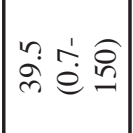 & 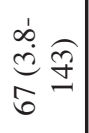 & 5 & $\stackrel{\text { I }}{I}$ & 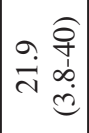 & वें & $\stackrel{\infty}{\infty} \underset{\sim}{\stackrel{d}{\Xi}}$ & $=$ & $\stackrel{\infty}{\stackrel{d}{d}} \underset{=}{=}$ & 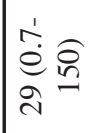 \\
\hline & 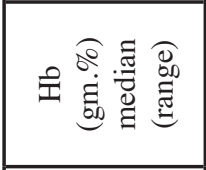 & $\stackrel{\frac{n}{n}}{\stackrel{n}{n}}$ & $\infty \underset{\infty}{\stackrel{\frac{n}{n}}{n}}$ & 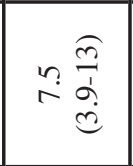 & $\approx \frac{\widehat{m}}{\dot{r}}$ & $\ddot{\sigma}$ & 9 & 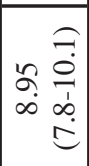 & 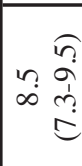 & 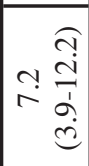 & $\stackrel{?}{?}$ & 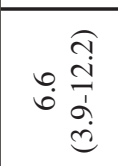 & $\begin{array}{l}\frac{\widehat{m}}{\frac{1}{d}} \\
\frac{a}{6}\end{array}$ \\
\hline & 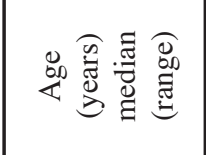 & 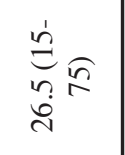 & $\begin{array}{l}\stackrel{1}{=} \\
\underset{n}{n}\end{array}$ & 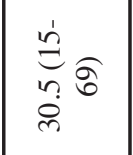 & $\begin{array}{l}\hat{\sigma} \\
\stackrel{0}{\sigma} \\
\stackrel{\sigma}{\sigma}\end{array}$ & 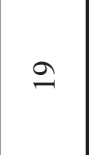 & 8 & 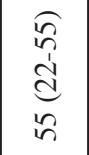 & 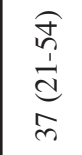 & 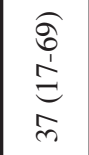 & $\vec{\lambda}$ & 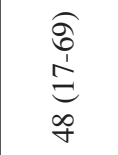 & 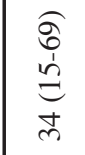 \\
\hline & 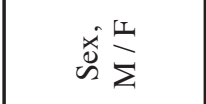 & 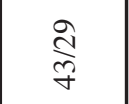 & $\stackrel{\infty}{0}$ & 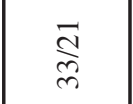 & $\frac{m}{\gamma}$ & $\bar{a}$ & $\stackrel{0}{=}$ & $\equiv$ & $\bar{\lambda}$ & 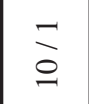 & $\stackrel{0}{=}$ & $\bar{a}$ & $\underset{\Xi}{\Xi}$ \\
\hline & $\frac{\widehat{d}}{z}$ & $\therefore 尺$ & $\begin{array}{c}\mathscr{d} \\
\stackrel{\infty}{-}\end{array}$ & $\begin{array}{l}5 \\
\frac{5}{5} \\
\text { in }\end{array}$ & $\underset{\hat{\theta}}{\stackrel{\sigma}{\sigma}}$ & $\underset{\underbrace{}}{\stackrel{f}{\Xi}}$ & $\underset{-}{\stackrel{f}{\rightleftarrows}}$ & $\underset{\substack{d \\
d}}{\stackrel{\alpha}{d}}$ & $\underset{m}{\stackrel{\overbrace =}{+}}$ & 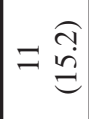 & $\stackrel{\overbrace{}}{\stackrel{f}{\rightleftarrows}}$ & $\circ \stackrel{\widehat{\infty}}{\stackrel{\theta}{=}}$ & 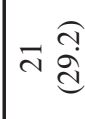 \\
\hline \multirow{6}{*}{ 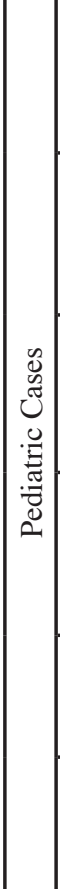 } & 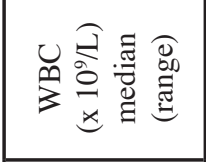 & 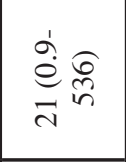 & 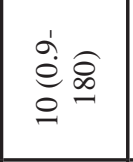 & 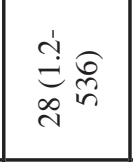 & 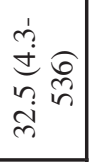 & 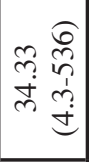 & 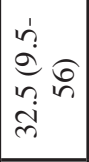 & & , & 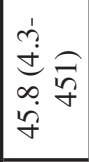 & $\stackrel{n}{y}$ & 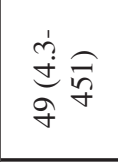 & 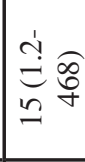 \\
\hline & 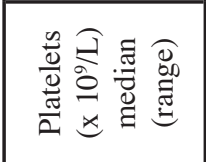 & 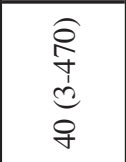 & 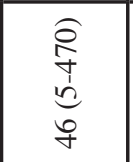 & $\begin{array}{l}\widehat{o} \\
\text { ì } \\
\text { ç } \\
\text { f }\end{array}$ & $\stackrel{1}{\stackrel{\overbrace{}}{\ominus}}$ & 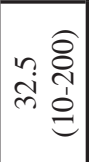 & 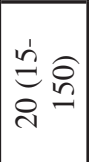 & & ' & ले $\frac{\hat{\sigma}}{\mathfrak{c}}$ & q & $\begin{array}{l}\widehat{\sigma} \\
\stackrel{n}{1} \\
\dot{c} \\
\text { aे }\end{array}$ & 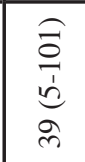 \\
\hline & 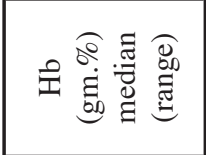 & 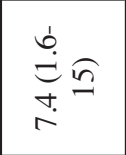 & 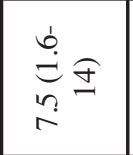 & $\begin{array}{l}\frac{\pi}{2} \\
\stackrel{d}{d} \\
\stackrel{n}{\sim}\end{array}$ & $\frac{\widehat{I}}{\frac{1}{1}}$ & $\begin{array}{ccc}2 & \widehat{7} \\
\infty & \frac{1}{0} \\
0 & 0\end{array}$ & $\begin{array}{l}\dot{n} \\
\dot{c} \\
n \\
n \\
r\end{array}$ & & ' & 色 & $r$ & $\begin{array}{l}\underset{J}{1} \\
b \\
\text { di } \\
0\end{array}$ & 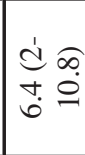 \\
\hline & 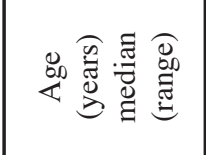 & $\underset{\forall}{\stackrel{1}{0}} \underset{\dot{\sigma}}{\dot{\theta}}$ & $\underset{\sigma}{\stackrel{1}{0}} \widehat{e}$ & 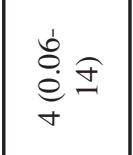 & 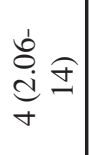 & $0 \underset{\frac{f}{d}}{\frac{f}{c}}$ & $\underset{m}{\stackrel{f}{d}}$ & & & 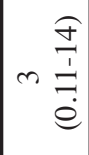 & $\infty$ & 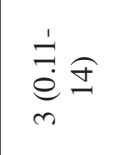 & 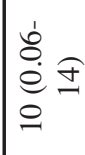 \\
\hline & 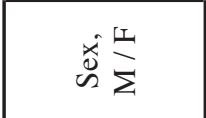 & $\frac{i}{\infty}$ & $\frac{\text { a }}{8}$ & 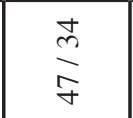 & $\stackrel{\Sigma}{\infty}$ & $\frac{n}{m}$ & $\frac{N}{i n}$ & & & $\stackrel{\infty}{ \pm}$ & $\stackrel{\circ}{=}$ & $\stackrel{\infty}{m}$ & $\frac{6}{r}$ \\
\hline & $\frac{\hat{\theta}}{z}$ & $\stackrel{\vartheta}{ \pm} \varrho$ & $\widetilde{\sigma} \underset{\dot{f}}{\stackrel{f}{Ð}}$ & $\bar{\infty} \underset{0}{0} \cdot$ & 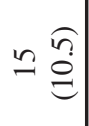 & $\begin{array}{l}6 \\
\dot{\infty} \\
\infty\end{array}$ & $\underset{r}{\stackrel{\partial}{d}}$ & & ' & 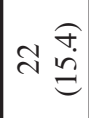 & $\underset{\mathfrak{e}}{\hat{e}}$ & 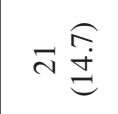 & $\underset{a}{a}$ \\
\hline \multirow{2}{*}{ 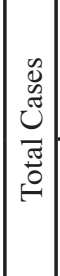 } & 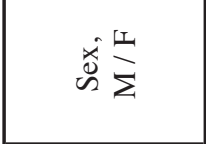 & $\begin{array}{l}n \\
\infty \\
\infty \\
\infty\end{array}$ & $\frac{i}{i n}$ & $\frac{n}{\infty}$ & $\frac{O}{\varrho}$ & $\frac{0}{m}$ & $\frac{1}{0}$ & $\equiv$ & $\bar{\lambda}$ & $\frac{a}{d}$ & $\stackrel{\circ}{\lambda}$ & $\frac{a}{\pi}$ & $\stackrel{\Xi}{\Xi}$ \\
\hline & $\frac{8}{z}$ & $\stackrel{n}{\sim} \underset{\varrho}{\sigma}$ & $\infty \underset{\frac{\hat{n}}{\hat{c}}}{\hat{c}}$ & $\begin{array}{l}\approx \\
\approx \\
\stackrel{\infty}{i}\end{array}$ & 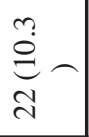 & $\underset{\sigma}{\stackrel{\overbrace + f}{d}}$ & 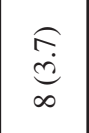 & $\underset{N}{\overparen{N}}$ & $\underset{m}{\stackrel{f}{\rightleftarrows}}$ & $m \stackrel{\tilde{n}}{\stackrel{n}{=}}$ & $\underset{\sim}{\Xi}$ & $\bar{m} \stackrel{\stackrel{f}{\stackrel{f}{巳}}}{ }$ & 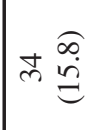 \\
\hline & 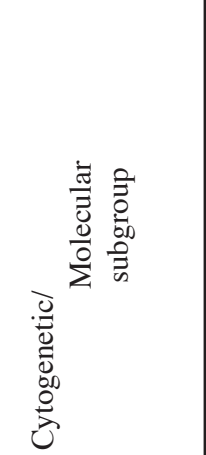 & 氶 & $\begin{array}{l}\overline{\tilde{E}} \\
\text { है } \\
\text { zे }\end{array}$ & 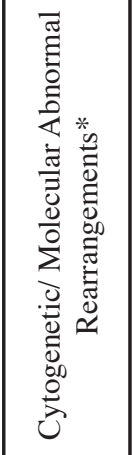 & 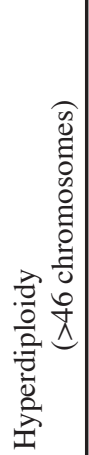 & 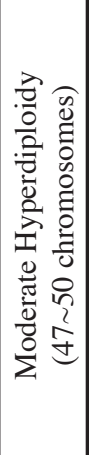 & 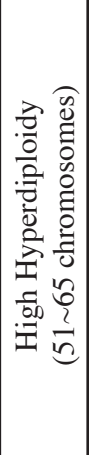 & 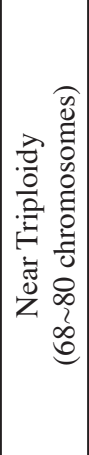 & 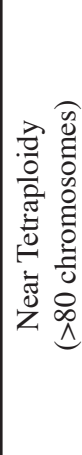 & 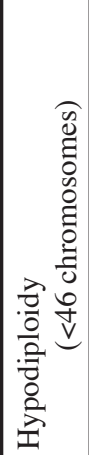 & 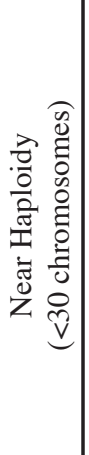 & 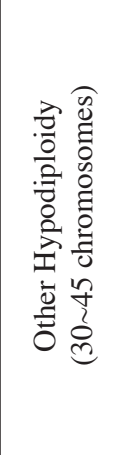 & 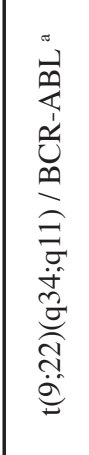 \\
\hline
\end{tabular}




\begin{tabular}{|c|c|c|c|c|c|c|c|c|c|c|c|c|c|c|c|c|c|c|c|c|c|c|c|}
\hline \multirow{6}{*}{ 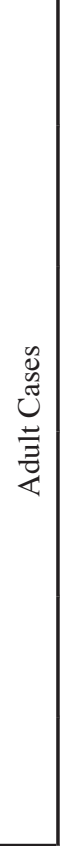 } & 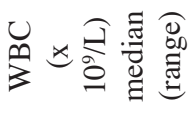 & 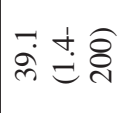 & $\sqrt[\curvearrowleft]{\forall}$ & ' & & ' & 1 & 1 & ' & ' & $\overrightarrow{\vec{i}}$ & $\begin{array}{l}\infty \\
0 \\
0\end{array}$ & $\hat{0}$ & ' & $\stackrel{n}{\beth}$ & 8 & & ' & & $\begin{array}{l}\stackrel{0}{2} \\
\stackrel{0}{n} \\
m\end{array}$ & $\vec{\sim}$ & 6 & 6 \\
\hline & 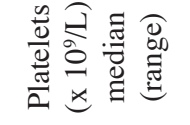 & $\underbrace{\infty}_{n} \underset{\infty}{\infty}$ & જે & ' & & ' & 1 & & & & $\approx$ & 6 & $=$ & 1 & $\stackrel{\sim}{\sim}$ & in & & ' & & $\check{\sim}$ & $\stackrel{n}{\wedge}$ & 9 & 9 \\
\hline & 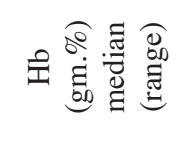 & $\begin{array}{l}\widehat{\sigma} \\
a \\
\mathfrak{c} \\
\end{array}$ & $\ddot{n}$ & ' & & ' & 1 & ' & & ' & 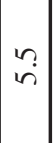 & $\stackrel{?}{r}$ & $\stackrel{m}{r}$ & ' & $\stackrel{\infty}{\infty}$ & 0 & & ' & ' & 0 & $n$ & $a$ & $a$ \\
\hline & 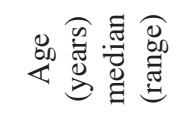 & 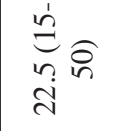 & こ & ' & & ' & 1 & 1 & & ' & 9 & $=$ & ป & 1 & ¿ & 8 & ' & ' & 1 & 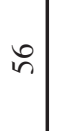 & $\cong$ & $\stackrel{\sim}{\sim}$ & $\stackrel{ \pm}{\sim}$ \\
\hline & 离立 & $\frac{\nabla}{\nabla}$ & $\bar{\partial}$ & ' & & ' & ' & 1 & ' & ' & $\stackrel{\circ}{=}$ & $\stackrel{0}{=}$ & $\stackrel{0}{-}$ & . & 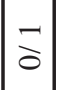 & $\stackrel{0}{-}$ & ' & ' & & $\grave{o}$ & $\stackrel{\circ}{=}$ & $\bar{\partial}$ & $\bar{\partial}$ \\
\hline & $\frac{\mathfrak{d}}{z}$ & $\underset{\infty}{\stackrel{\overbrace{}}{\Xi}}$ & $\underset{=}{\stackrel{f}{\Xi}}$ & ' & & ' & 1 & ' & & ' & 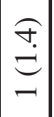 & $\underset{\overbrace{}}{\stackrel{f}{\rightleftarrows}}$ & 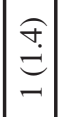 & ' & 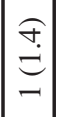 & 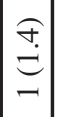 & & ' & & $\underset{Ð}{\stackrel{f}{\Xi}}$ & $\underset{\sim}{\stackrel{f}{\Xi}}$ & 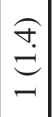 & $\underset{\sim}{\stackrel{f}{\Xi}}$ \\
\hline \multirow{6}{*}{ 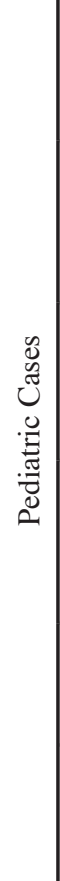 } & 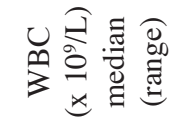 & $\begin{array}{l}\frac{\widehat{o}}{8} \\
\frac{1}{b} \\
o \\
o\end{array}$ & 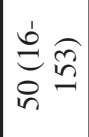 & 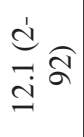 & & $N$ & $\vec{r}$ & $\stackrel{?}{+}$ & $\frac{9}{6}$ & $\stackrel{n}{+}$ & 1 & 1 & 1 & $\sim$ & 1 & 1 & $\stackrel{?}{+}$ & $\stackrel{\nabla}{\vec{\nu}}$ & $\stackrel{\vartheta}{\forall}$ & ' & 1 & 1 & 1 \\
\hline & 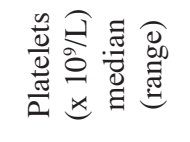 & 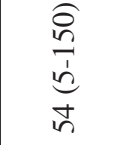 & $\begin{array}{l}\text { } \\
\hat{1} \\
e \\
i n\end{array}$ & $\begin{array}{l}\stackrel{1}{\ominus} \\
n \\
n \\
n \\
n\end{array}$ & & 요 & 2 & $\nexists$ & 点 & $n$ & ' & 1 & ' & ஓ & 1 & 1 & ㄱ. & $m$ & ભે & & 1 & ' & 1 \\
\hline & 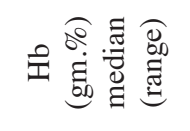 & $\underset{\substack{j \\
\dot{j}}}{\stackrel{1}{-}}$ & 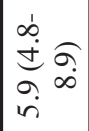 & $\begin{array}{l}\frac{\sigma}{n} \\
\frac{1}{6} \\
a \\
a\end{array}$ & & $\stackrel{n}{\sim}$ & $\stackrel{n}{+}$ & $\begin{array}{l}0 \\
\infty\end{array}$ & $\stackrel{\infty}{\sim}$ & $=$ & ' & 1 & ' & 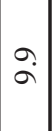 & 1 & ' & $\begin{array}{l}0 \\
\infty\end{array} \mid$ & $\stackrel{\Upsilon}{\nearrow}$ & $\stackrel{9}{=}$ & ' & 1 & 1 & 1 \\
\hline & 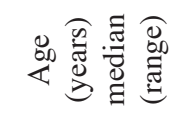 & $\begin{array}{l}\frac{\pi}{1} \\
\stackrel{n}{n}\end{array}$ & $\frac{a}{d}$ & 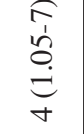 & & 0 & 0 & $\simeq$ & in & $m$ & 1 & 1 & 1 & $\sim$ & ' & ' & $\simeq$ & $n$ & - & ' & 1 & 1 & ' \\
\hline & 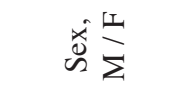 & $\frac{ \pm}{a}$ & $\frac{N}{m}$ & $\frac{m}{n}$ & & $\succsim$ & $\stackrel{\circ}{-}$ & 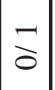 & $\bar{\partial}$ & $\stackrel{0}{=}$ & ' & 1 & ' & 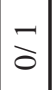 & 1 & ' & $\bar{\partial}$ & $\stackrel{\circ}{=}$ & $\bar{o}$ & ' & 1 & 1 & 1 \\
\hline & $\frac{\widehat{d}}{z}$ & $\begin{array}{l}\overparen{a} \\
\stackrel{9}{2}\end{array}$ & 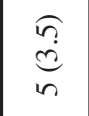 & $\begin{array}{l}6 \\
\infty \\
\infty \\
\infty\end{array}$ & & $\begin{array}{c}\hat{\imath} \\
\dot{e} \\
-\end{array}$ & $\begin{array}{c}\hat{E} \\
\dot{e} \\
-\end{array}$ & $\begin{array}{c}\hat{E} \\
e \\
-\end{array}$ & $\begin{array}{c}\hat{E} \\
e \\
-\end{array}$ & $\begin{array}{c}\hat{E} \\
\hat{e} \\
-\end{array}$ & 1 & 1 & ' & $\begin{array}{c}\hat{E} \\
e \\
-\end{array}$ & 1 & , & $\begin{array}{l}\hat{E} \\
e \\
-\end{array}$ & $\begin{array}{c}\hat{E} \\
e \\
-\end{array}$ & $\begin{array}{c}\hat{E} \\
\stackrel{e}{c}\end{array}$ & ' & 1 & 1 & , \\
\hline \multirow{2}{*}{ 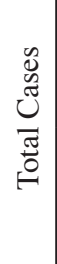 } & $\stackrel{\dot{x}}{\tilde{D}} \sum$ & $\frac{\infty}{2}$ & $\frac{m}{m}$ & $\frac{m}{n}$ & & 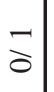 & $\stackrel{\circ}{=}$ & $\bar{\partial}$ & $\bar{\partial}$ & $\stackrel{0}{=}$ & $\stackrel{\circ}{-}$ & $\stackrel{0}{=}$ & $\stackrel{0}{=}$ & - & $\overline{0}$ & $\stackrel{0}{=}$ & $\overline{0}$ & $\stackrel{\circ}{=}$ & $\bar{\partial}$ & $\bar{\partial}$ & $\stackrel{\circ}{-}$ & $\bar{\partial}$ & $\bar{\partial}$ \\
\hline & $\frac{8}{z}$ & $\begin{array}{l}\widehat{\infty} \\
\stackrel{0}{ } \\
\vec{\sim}\end{array}$ & $\begin{array}{l}\text { ô } \\
\text { di } \\
0\end{array}$ & $\underset{\infty}{\stackrel{0}{\infty}}$ & & 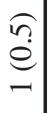 & $\begin{array}{c}n \\
\tilde{n} \\
e \\
-1\end{array}$ & $\begin{array}{c}\mathfrak{n} \\
e \\
e \\
-\end{array}$ & $\begin{array}{c}\tilde{c} \\
\mathfrak{n} \\
- \\
-\end{array}$ & $\begin{array}{c}0 \\
n \\
e \\
-1\end{array}$ & $\begin{array}{c}\mathfrak{n} \\
e \\
e \\
-\end{array}$ & $\begin{array}{c}n \\
n \\
e \\
-1\end{array}$ & $\begin{array}{c}\tilde{n} \\
e \\
- \\
-\end{array}$ & $\begin{array}{c}\tilde{n} \\
e \\
- \\
-1\end{array}$ & $\mid \begin{array}{c}n \\
n \\
e \\
-1\end{array}$ & $\begin{array}{c}\tilde{n} \\
e \\
-\end{array}$ & $\begin{array}{c}\tilde{n} \\
e \\
-1\end{array}$ & $\begin{array}{c}\tilde{n} \\
e \\
- \\
-\end{array}$ & $\begin{array}{c}\mathfrak{n} \\
e \\
-\end{array}$ & $\begin{array}{c}\mathfrak{n} \\
e \\
-1\end{array}$ & 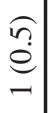 & $\begin{array}{c}\tilde{n} \\
e \\
- \\
-1\end{array}$ & $\begin{array}{c}\widehat{n} \\
e \\
- \\
-\end{array}$ \\
\hline & 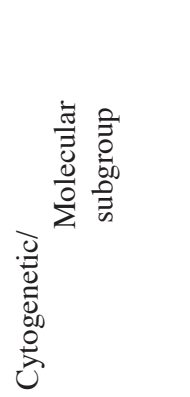 & 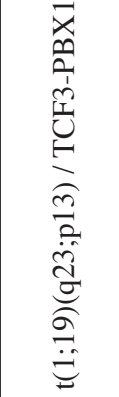 & 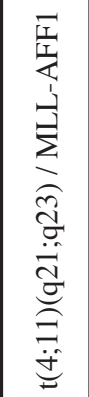 & 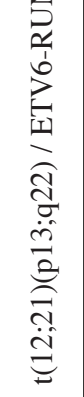 & 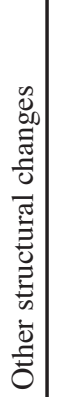 & 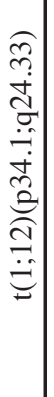 & 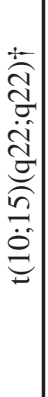 & 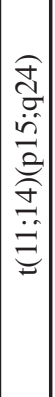 & 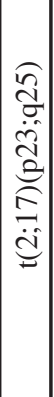 & 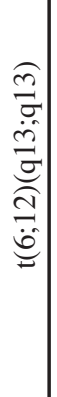 & 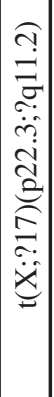 & 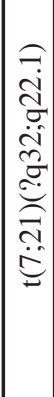 & 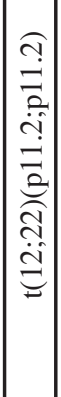 & 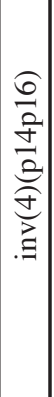 & 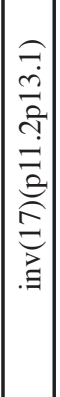 & 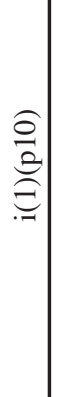 & 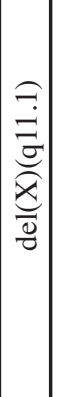 & 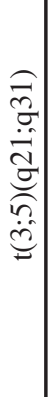 & 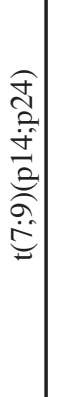 & 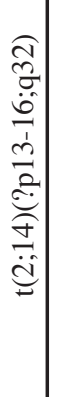 & 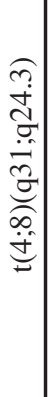 & 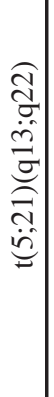 & 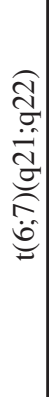 \\
\hline
\end{tabular}




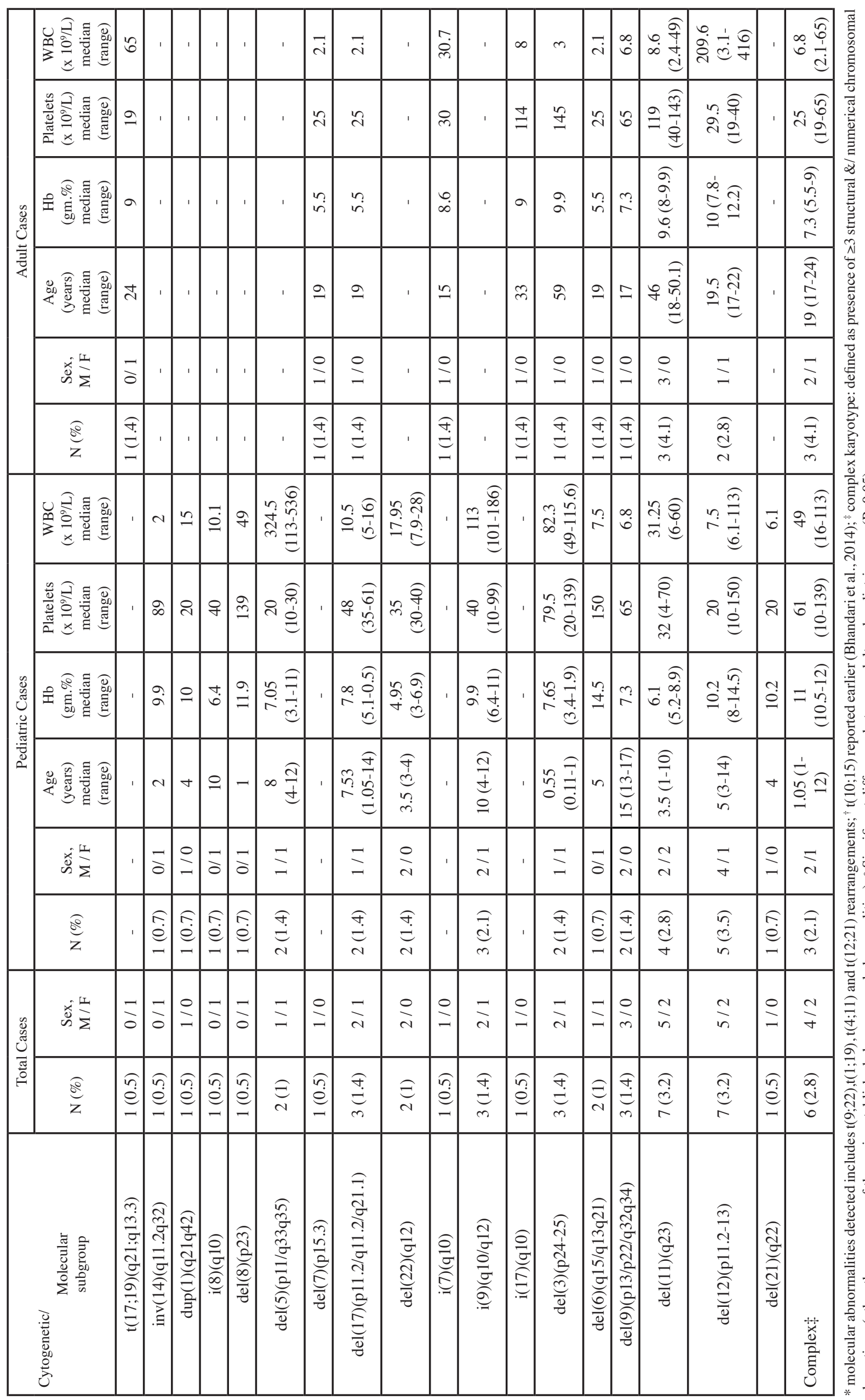


Table 3. Age-Sex-Modal Number Distribution Pattern in Various Indian B-lineage ALL Patients

\begin{tabular}{|c|c|c|c|c|c|c|c|c|c|c|c|c|c|c|}
\hline \multirow{3}{*}{$\begin{array}{l}\text { Type of } \\
\text { Ploidy } \\
\text { change } \\
\text { (Modal No.) }\end{array}$} & \multicolumn{4}{|c|}{ Pediatric Patients } & \multicolumn{4}{|c|}{ Adult Patients } & \multicolumn{4}{|c|}{ Total Patients } & \multirow{3}{*}{$\begin{array}{c}\text { Males } \\
(\mathrm{T}=130) \\
\mathrm{N}(\%)\end{array}$} & \multirow{3}{*}{$\begin{array}{c}\text { Females } \\
(\mathrm{T}=85) \\
\mathrm{N}(\%)\end{array}$} \\
\hline & \multirow{2}{*}{$\frac{(\mathrm{T}=143)}{\mathrm{N}(\%)}$} & \multicolumn{2}{|c|}{ Sex } & \multirow{2}{*}{$\begin{array}{c}\text { Age } \\
\text { Range }\end{array}$} & \multirow{2}{*}{$\begin{array}{l}(\mathrm{T}=72) \\
\mathrm{N}(\%)\end{array}$} & \multicolumn{2}{|c|}{ Sex } & \multirow{2}{*}{$\begin{array}{c}\text { Age } \\
\text { Range }\end{array}$} & \multirow{2}{*}{\begin{tabular}{|c|}
$(\mathrm{T}=215)$ \\
$\mathrm{N}(\%)$ \\
\end{tabular}} & \multicolumn{2}{|c|}{ Sex } & \multirow{2}{*}{$\begin{array}{l}\text { Age } \\
\text { Range }\end{array}$} & & \\
\hline & & $M$ & $\mathrm{~F}$ & & & $\mathrm{M}$ & $\mathrm{F}$ & & & $M$ & $\mathrm{~F}$ & & & \\
\hline $\begin{array}{c}\text { Hypo } \\
(2 n \leq 23-29)\end{array}$ & $1(0.7)$ & 1 & 0 & 8 & $1(1.4)$ & 1 & 0 & 23 & $2(1)$ & 2 & 0 & $8-23$ & $2(1.5)$ & $0(0)$ \\
\hline $\begin{array}{c}\text { Hypo } \\
(2 \mathrm{n}=30-39)\end{array}$ & $10(7)$ & 8 & 2 & $0.11-8$ & $3(4.2)$ & 3 & 0 & $17-48$ & $13(6)$ & 11 & 2 & $0.11-48$ & $11(8.5)$ & $2(2.35)$ \\
\hline $\begin{array}{c}\text { Нypo } \\
(2 \mathrm{n}=40-45)\end{array}$ & $11(7.7)$ & 5 & 6 & $1-14$ & $6(9.7)$ & 5 & 1 & $33-69$ & $18(8.4)$ & 11 & 7 & $1-69$ & $11(8.5)$ & $7(8.2)$ \\
\hline $\begin{array}{l}\text { Normal } \\
(2 n=46) \\
\end{array}$ & $62(43)$ & 40 & 22 & $0.01-13$ & $18(25)$ & 10 & 8 & $15-75$ & $\begin{array}{c}80 \\
(37.2) \\
\end{array}$ & 50 & 30 & $0.01-75$ & $\begin{array}{c}50 \\
(38.5) \\
\end{array}$ & $\begin{array}{c}30 \\
(35.3) \\
\end{array}$ \\
\hline $\begin{array}{c}\text { Hyper } \\
(2 \mathrm{n}=47-50)\end{array}$ & $8(5.6)$ & 3 & 5 & $3-14$ & $1(1.4)$ & 0 & 1 & 19 & $9(4.2)$ & 3 & 6 & $3-19$ & $3(2.3)$ & $6(7)$ \\
\hline $\begin{array}{c}\text { Hyper } \\
(2 \mathrm{n}=51-65)\end{array}$ & $7(4.9)$ & 5 & 2 & $2-4$ & $1(1.4)$ & 1 & 0 & 66 & $8(3.7)$ & 6 & 2 & $2-66$ & $6(4)$ & $2(2.35)$ \\
\hline $\begin{array}{c}\text { Hyper } \\
(2 \mathrm{n}=66-80)\end{array}$ & $0(0)$ & 0 & 0 & 0 & $2(2.8)$ & 1 & 1 & $22-55$ & $2(1)$ & 1 & 1 & $22-55$ & $1(0.77)$ & $1(1.17)$ \\
\hline $\begin{array}{l}\text { Hyper }(2 \mathrm{n} \\
\geq 81)\end{array}$ & $0(0)$ & 0 & 0 & 0 & $2(4.2)$ & 2 & 0 & $21-54$ & $3(1.4)$ & 2 & 1 & $21-54$ & $2(1.5)$ & $1(1.17)$ \\
\hline
\end{tabular}

Table 4. Comparison of Chromosomal Aberrations between Indian and Western Pediatric ALL Patients

\begin{tabular}{|c|c|c|c|c|c|c|c|c|}
\hline Study & Country & $\begin{array}{c}\text { Total } \\
\text { cases, } \\
\mathrm{n}\end{array}$ & $\begin{array}{l}\text { Hyperdiploidy, } \\
\mathrm{n}(\%)\end{array}$ & $\begin{array}{l}\text { Hypodiploidy, } \\
\text { n (\%) }\end{array}$ & $\begin{array}{l}\mathrm{t}(9 ; 22) / \\
\mathrm{BCR}- \\
\mathrm{ABL} \\
\mathrm{n}(\%)\end{array}$ & $\begin{array}{c}\mathrm{t}(1 ; 19) / \\
\mathrm{TCF} 3- \\
\text { PBX1, } \\
\mathrm{n}(\%)\end{array}$ & $\begin{array}{l}\mathrm{t}(4 ; 11) / \\
\text { MLL- } \\
\text { AFF1, } \\
\mathrm{n}(\%)\end{array}$ & \begin{tabular}{|l}
$\mathrm{t}(12 ; 21) /$ \\
ETV6- \\
RUNX1, \\
$\mathrm{n}(\%)$
\end{tabular} \\
\hline Present Study, 2015 & India & 143 & $15(10.5)$ & $22(15.4)$ & $13(9.1)$ & $13(9.1)$ & $5(3.5)$ & $8(5.6)$ \\
\hline Fauzdar et al. 2010 & India & 77 & $21(27)^{a}$ & $4(5)^{a}$ & $9(10)$ & - & - & $9 / 21(42)^{a}$ \\
\hline Hill et al. 2005 & India & 42 & - & - & - & 0 & 0 & $2(4.8)$ \\
\hline Sazawal et al. 2004 & India & 35 & - & - & $1(2.8)$ & $2(5.7)$ & 0 & 0 \\
\hline Siraj et al. 2003 & India & 259 & - & - & $14(5)$ & $18(7)$ & 0 & $18(7)$ \\
\hline Safaei et al. 2013 & Iran & 88 & $28(31.8)^{a, b}$ & $4(4.5)^{a}$ & $1(1.1)^{\mathrm{a}}$ & $1(1.1)^{\mathrm{a}}$ & - & 0 \\
\hline Chen et al. 2012 & China & 726 & $77(10.6)^{b}$ & - & $\begin{array}{c}106 \\
(14.6) \\
\end{array}$ & $39(5.3)$ & $20(2.7)$ & $\begin{array}{l}83 / 541 \\
(15.3)^{a}\end{array}$ \\
\hline Alonso et al. 2012 & Argentina & 326 & $63(20)^{\mathrm{a}}$ & $6(1.8)^{a}$ & $6(1.8)^{\mathrm{a}}$ & $19(5.8)$ & $38(11.6)^{\text {a }}$ & $49(15)^{\mathrm{a}}$ \\
\hline $\begin{array}{c}\text { DeBraekeleer et al. } \\
2010\end{array}$ & France & 93 & $37(39)^{a, b}$ & $4(4.3)^{\mathrm{a}}$ & $4(4.3)$ & $6(6.5)$ & $2(2.1)$ & $13(14)^{a}$ \\
\hline $\begin{array}{c}\text { Moorman et al. } \\
2010 \\
\end{array}$ & Britain & 1725 & $562(38)^{a, b}$ & $18(1)^{a}$ & $43(3)^{a}$ & $50(4)^{a}$ & $17(0.98)^{\mathrm{a}}$ & $368(25)^{\mathrm{a}}$ \\
\hline Mesquita et al. 2009 & Brazil & 88 & $11(12.5)$ & $4(4.5)^{a}$ & $(3.03)$ & $(9.68)$ & 0 & $(21.21)^{\mathrm{a}}$ \\
\hline Ceppi et al. 2009 & \begin{tabular}{|l|} 
Nicaragua \\
\end{tabular} & 64 & $16(25)^{a, b}$ & - & $1(1.5)^{\mathrm{a}}$ & - & $2(3.1)$ & $9(14)^{a}$ \\
\hline $\begin{array}{c}\text { Santamaria-Quesada } \\
\text { et al. } 2009\end{array}$ & Costa Rica & 65 & $15(23)^{a}$ & $1(1.5)^{\mathrm{a}}$ & $3(4.6)$ & $2(3)$ & $1(1.5)$ & $12(18.4)^{a}$ \\
\hline Hann et al. 2001 & Britain & 1658 & $566(34)^{a}$ & $109(6.5)^{a}$ & $25(1.5)^{\mathrm{a}}$ & $47(2.8)^{\mathrm{a}}$ & $15(0.90)^{\mathrm{a}}$ & $\begin{array}{l}128 / 659 \\
(19.4)^{a} \\
\end{array}$ \\
\hline Gaynon et al. 2000 & USA & 1946 & $494(25.4)^{a, b}$ & $114(5.8)^{\mathrm{a}}$ & $44(2.3)^{\mathrm{a}}$ & $67(3.4)^{\mathrm{a}}$ & $42(2.2)$ & $\begin{array}{l}95 / 504 \\
(18.8)^{a}\end{array}$ \\
\hline
\end{tabular}

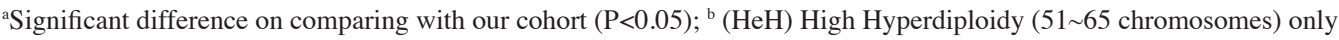

Besides, common alterations observed varied between $0.5-5 \%$ cases while 6 patients showed complex karyotypes (Table 2).

Additionally as shown in Figure 2, in the current study FISH analysis helped reveal additional structural or numerical changes. These included cryptic deletions at ABL breakpoint on chromosome 9(q) (n=7), multiplication involving the 22q11.2 BCR locus $(n=1)$, presence of masked double/ multiple Philadelphia chromosome $(n=4)$ in BCR-ABL positive patients; and deletions of non- 
Table 5. Comparison of Chromosomal Aberrations between Indian and Western Adult ALL Patients

\begin{tabular}{|c|c|c|c|c|c|c|c|c|}
\hline Study & Country & $\begin{array}{c}\text { Total } \\
\text { cases, } \\
\mathrm{n}\end{array}$ & $\begin{array}{c}\text { Hyperdiploidy, } \\
\mathrm{n}(\%)\end{array}$ & $\begin{array}{c}\text { Hypodiploidy, } \\
\mathrm{n}(\%)\end{array}$ & $\begin{array}{c}\mathrm{t}(9 ; 2) / \mathrm{BCR}- \\
\text { ABL, }(\%)\end{array}$ & $\begin{array}{c}\mathrm{t}(1 ; 19) / \\
\text { TCF3- } \\
\text { PBX1, } \mathrm{n} \\
(\%)\end{array}$ & $\begin{array}{c}\mathrm{t}(4 ; 11) / \\
\text { MLL- } \\
\text { AFF1, } \mathrm{n} \\
(\%)\end{array}$ & $\begin{array}{c}\mathrm{t}(12 ; 21) / \\
\text { ETV6- } \\
\text { RUNX1, } \\
(\%)\end{array}$ \\
\hline $\begin{array}{c}\text { Present } \\
\text { Study, 2015 }\end{array}$ & India & 72 & $7(9.7)$ & $11(15.2)$ & $21(29.2)$ & $8(11.1)$ & $1(1.4)$ & 0 \\
\hline $\begin{array}{c}\text { Sazawal et } \\
\text { al. 2004 }\end{array}$ & India & 34 & - & - & $5(14.7)$ & $2(5.8)$ & 0 & 0 \\
\hline $\begin{array}{c}\text { Safaei et al. } \\
2013\end{array}$ & Iran & 40 & $3(7.5)^{\mathrm{b}}$ & $1(2.5)^{\mathrm{a}}$ & $7(17.5)$ & $1(2.5)$ & - & 0 \\
\hline $\begin{array}{c}\text { Chen et al. } \\
2012\end{array}$ & China & 296 & $7(2.3)^{\mathrm{a}} \mathrm{b}$ & - & $106(35.8)$ & $2(0.6)^{\mathrm{a}}$ & $21(7)$ & $2 / 241(0.8)$ \\
\hline $\begin{array}{c}\text { Moorman et } \\
\text { al. 2010 }\end{array}$ & Britain & 240 & $17(7)^{\mathrm{b}}$ & $7(3)^{\mathrm{a}, \mathrm{c}}$ & $32 / 208(15.3)^{\mathrm{a}}$ & $(<10)^{\mathrm{a}}$ & $(<10)$ & - \\
\hline $\begin{array}{c}\text { DeBraekeleer } \\
\text { et al. 2010 }\end{array}$ & France & 84 & $9(10.7)^{\mathrm{b}}$ & $13(15.47)$ & $28(33.3)$ & $1(1.2)^{\mathrm{a}}$ & $6(7.1)$ & 0 \\
\hline $\begin{array}{c}\text { Mancini et } \\
\text { al. 2005 }\end{array}$ & Italy & 266 & $18(6.77)^{\mathrm{b}}$ & - & $101(37.9)$ & $7(2.6)^{\mathrm{a}}$ & $24(9)^{\mathrm{a}}$ & $1 / 162(0.6)$ \\
\hline $\begin{array}{c}\text { Secker et al. } \\
1997\end{array}$ & Britain & 204 & $24(12)^{\mathrm{b}}$ & $8(4)^{\mathrm{a}}$ & $33(16)^{\mathrm{a}}$ & $7(3)^{\mathrm{a}}$ & $3(1)$ & - \\
\hline $\begin{array}{c}\text { GFCH, 1996 } \\
\text { Grance }\end{array}$ & 339 & $30(8.8)^{\mathrm{b}}$ & $34(10)$ & $127(37.4)$ & $11(3.2)^{\mathrm{a}}$ & $16(4.7)$ & - \\
\hline $\begin{array}{c}\text { Walters et al. } \\
1990\end{array}$ & USA & 103 & $(12)$ & $(2)^{\mathrm{a}}$ & $(12.6)^{\mathrm{a}}$ & - & - & - \\
\hline
\end{tabular}

${ }^{\text {a }}$ Significant difference on comparing with our cohort $(\mathrm{P}<0.05) ;{ }^{\mathrm{b}}$ (HeH)-High Hyperdiploidy $\left(51 \sim 65\right.$ chromosomes) only; ${ }^{\mathrm{c}}$ Low hyperdiploidy (30-39 chromosomes) / Near triploidy (60-78 chromosomes).

translocated ETV6 allele from 12p13 (n=2), deletions of residual RUNX1 allele on chromosome $21(\mathrm{n}=2)$ and multiplication involving the 21 RUNX1 locus $(n=1)$ in cases suspected of ETV6-RUNX1. The novel abnormality of $\mathrm{t}(10 ; 15)(\mathrm{q} 22 ; \mathrm{q} 22)$ has previously been reported by our group (Bhandari et al., 2014). Further, in the present study molecular [FISH and RT-PCR] techniques helped overcome hurdles of detection of cryptic rearrangements and lack of metaphase cells allowing identification of $\mathrm{t}(9 ; 22)(\mathrm{n}=24), \mathrm{t}(12 ; 21)(\mathrm{n}=8), \mathrm{t}(1 ; 19)(\mathrm{n}=16)$ and $11 \mathrm{q} 23$ rearrangements $(n=5)$. In the present series, there was a patient in which two different fusion oncogenes $t(9 ; 22) /$ BCR-ABL and $\mathrm{t}(1 ; 19) / \mathrm{TCF} 3-\mathrm{PBX} 1$ were confirmed by RT-PCR analysis although its karyotype was unsuccessful.

\section{Discussion}

The current study bears similarities with several ALL reports in certain aspects- viz. preponderance of ALL in males $(60.5 \%$ observed $v s$. previously reported 60-85\%) (Sazawal et al., 2004; Fauzdar et al., 2010; Chen et al., 2012); higher frequency of ALL in children (66.5\% observed $v s$. previously reported $70 \%$ ) (Chen et al., 2012; Safaei et al., 2013); preponderance of WBC counts less than $50,000 / \mathrm{mm}^{3}(71.6 \%$ observed $v s$. previously reported $72-77 \%$ ) (Siraj et al., 2003; Chen et al., 2012); karyotypic culture success rate ( $80 \%$ observed vs. previously reported $83 \%$ ) (Alonso et al., 2012) and higher frequency of chromosomal abnormalities $(62.8 \%$ observed vs. previously reported 61.8\%) (SantamariaQuesada et al., 2009).

Although several molecular cytogenetic techniques allow detection of chromosomal aberrations, conventional karyotyping still is the gold standard as it provides a global overview of the genome and a baseline to trace the evolution of the disease. To the best of our knowledge, this is one of the largest reported series of cytogenetic investigation in Indian B-lineage ALL cases presenting prevalence of each cytogenetic subtype listed in WHO classification.

\section{Distributions of chromosomal rearrangements}

In the current study, we compared frequencies of major cytogenetic subtypes between different groups and our series for pediatric and adult ALL, respectively (Tables 4 and 5). Considering the potential ethnic background difference between Western and Indian populations, such kind of investigation is important for Indian ALL patients.

As seen in Tables 4 and 5, Hypodiploidy was the most common abnormality with a high frequency in our pediatric $(15.4 \%$ vs $1-6.5 \%$, all $\mathrm{P}<0.05)$ and adult (15.2\% vs $1.9-3.9 \%$, all $\mathrm{P}<0.05)$ series. Several reports from France $(15.4 \%, 10 \%)$ show concordance with our adult results (GFCH, 1996; Braekeleer et al., 2010) (Table 5). Interestingly, Amare et al. From India reported further enhanced incidences that were two fold higher or even greater (44.4\% in adults and $38.4 \%$ in pediatrics) (Amare et al., 1999). These findings specify the fact of relatively high frequency of hypodiploidy in India suggesting that geographical and ethnic factors cannot be excluded. The scientific significance of hypodiploidy in ALL is its association with various clinical outcomes. A progressively worse outcome is reported with decreasing chromosome number, with an approximate 6-year EFS 
of $65 \%, 40 \%$ and $25 \%$ for patients with $45,33-44$ and $<28$ chromosomes respectively (Heerema et al., 1999). Notably, near-haploidy and low-hypodiploidy are rare subgroups, associated with very poor outcome. Often they go underdiagnosed when masked by a co-existing good-prognostic hyperdiploid (>50 chromosomes) line (Stark et al., 2001). In the present study, two male patients revealed near-haploid karyotypes with various levels of ploidy ranging from near-haploidy (21 chromosomes) to hypodiploidy (45 chromosomes) to near-triploidy (60 chromosomes) to near-tetraploidy (90 chromosomes).

Interestingly a higher frequency of $\mathrm{t}(1 ; 19)$ was observed in both our pediatric $(9.1 \%$ vs $1-3.4 \%$, all $\mathrm{P}<0.05)$ and adult $(11.1 \%$ vs $0.6-3.2 \%$, all $\mathrm{P}<0.05)$ groups (Tables 4 and 5). Notably, similar results in childhoodB-ALL were reported from Brazil (9.68\%) (Mesquita et al., 2009) and Mexico (11.5\%) (Jimenez-Morales et al., 2008). While $t(1 ; 19)$ is associated with nearly good outcome with modern intensive protocols, $t(9 ; 22)$ cases are associated with extremely poor prognosis and high relapse rates; hence, early detection is utterly crucial to initiate more aggressive therapy. In our adult ALL series, $t(9 ; 22)$ frequency was in agreement with most reports $(29.2 \% \mathrm{vs}$ $33-37 \%)$ and higher than few countries (29.2\% vs $12.6-$ $19 \%$, all $\mathrm{P}<0.05$ ) (Table 5). Likewise high frequency of $\mathrm{t}(9 ; 22)$ was observed in pediatric cases $(9.1 \%$ vs $1.1-3 \%$, all $\mathrm{P}<0.05$ ) (Table 4). Strikingly, several Asian studies showed a similar trend for $\mathrm{t}(9 ; 22)$ in childhood-ALL (Pakistan-44\%; India-12\%,15\%) (Awan et al., 2012; Mazloumi et al., 2012; Pandita et al., 2015) and adultALL (India-25\%; Pakistan-20\%) (Bhatia et al., 2012; Sabir et al., 2012).

The $\mathrm{t}(12 ; 21)$ subtype was absent in our adult series, in agreement with previous reports (Table 5 ). In our pediatric series, $\mathrm{t}(12 ; 21)$ was lower than most reports $(5.6 \% \mathrm{vs}$. $14.2-42 \%$, all $\mathrm{P}<0.05$ ) (Table 4 ), yet similar to previous Indian studies (4.3-7\%) (Siraj et al., 2003; Hill et al., 2005; Mazloumi et al., 2012). Likewise, $t(4 ; 11)$ was less frequent in our study, in agreement with previous reports for this sub-group (Table 4 and 5). Exceptions to our pediatric and adult results $(3.5 \%, 1.4 \%)$ included lower frequencies from UK $(0.98 \%, 0.9 \%)$ (Hann et al., 2001; Moorman et al., 2010a) and higher frequencies from Argentina (11.6\%) (Alonso et al., 2012), Italy (9\%) (Mancini et al., 2005) (all $\mathrm{P}<0.05)$ (Table 4 and 5). Previous Asian studies have associated $\mathrm{t}(12 ; 21)$ patients with favorable clinical outcome and $\mathrm{t}(4 ; 11)$ patients with poor outcome; hence, identifying these prognostic markers aids in determining the risk and further treatment (Amare et al., 1999; Sabir et al., 2012).

In contrast, hyperdiploidy in our pediatric cases was significantly lower than few Asian (Fauzdar et al., 2010; Safaei et al., 2013) and most western countries (10.5\% vs 25-39\%, all $\mathrm{P}<0.05$ ) (Table 4). Strikingly, similar reports of lower hyperdiploidy in childhood-ALL are observed in India $(5 \% ; 14.8 \% ; 15 \%$ ) (Amare et al., 1999; Mazloumi et al., 2012; Pandita et al., 2015), China (10.6\%) (Chen et al., 2012), Brazil (12.5\%) (Mesquita et al., 2009) and Pakistan (13.4\%) (Shaikh et al., 2014). Also, gain of chromosomes $2,5,6,8,9,10,21,22(\mathrm{n}=2,9.1 \%)$ and $1,3,7,11,12,14$, $15,16(\mathrm{n}=1,4.5 \%)$ were observed in pediatric hyperdiploid
Chromosomal Aberrations in Indian B Lineage ALL Patients cases. Hyperdiploidy in our adult series $(9.7 \%)$ was similar to most reports, yet exceptionally higher than $2.3 \%$ of China (Chen et al., 2012) $(\mathrm{P}<0.05)$ (Table 5). Response to treatment rates varies among chromosome ploidy groups. Hyperdiploid-cases are associated with favorable outcome and less intense therapy (5-year EFS $>84 \%$ ), probably owing to increased accumulation of anti-leukemic drugs (methotrexate) in hyperdiploid blast-cells (Moorman et al., 2010a). Near-triploidy occurs rarely in childhood ALL (1\%), and represents only 3-5\% of the adult cases $(\mathrm{GFCH}$, 1996; Secker-Walker et al., 1997). In present study, similar results were observed as triploidy and tetraploidy were absent in the pediatric cases and represented $3 \%$ adult cases. Thus in contrast to western reports, our study highlights ploidy variability such as scarcity of hyperdiploids and preponderance of hypodiploids.

Conventional cytogenetics, interphase FISH and multiplex RT-PCR measure chromosomal abnormalities at different biological levels. Cytogenetics involves analysis of metaphases and hence results are based only on the dividing leukemic cells. Whereas FISH and RT-PCR can determine rearrangements independent of cell division. Thus failure to identify chromosomal rearrangements cytogenetically may be attributed (a) to the presence of low levels of non-dividing leukemic cells (containing the chromosomal rearrangements) in the sample (Campbell et al., 1999; Watanabe et al., 2003); and (b) to the more sensitive molecular techniques like RT-PCR (detection

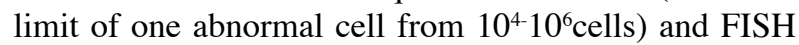
(detection limit of $10^{3}$ cells) (Polampalli et al., 2011).

The contribution of novel chromosomal rearrangements in B-Lineage ALL is less clear and few have been mapped in detail in the Indian sub-continent. All such abnormalities detected in the present study were checked against database of chromosomal aberration [http://atlasgeneticsoncology. org/]. Although the exact consequences of such rare, novel aberrations remain to be elucidated, they could represent an important event in the tumorigenesis of hematological malignancies.

Certain common changes observed included del(12) (p11.2-13) (3.2\%), del(11q23) (3.2\%), del(6)(q15/q13q21) $(1 \%), \operatorname{del}(3)(\mathrm{p} 24-25)(1.4 \%), \operatorname{del}(9)(\mathrm{p} 13 / \mathrm{p} 22 / \mathrm{q} 32 \mathrm{q} 34)$ (1.4\%), i(7)(q10) (0.5\%), i(9)(q10/q12) (1.4\%) and i(17) (q10) $(0.5 \%)$. In childhood ALL, all 12p cytogenetic abnormalities do not involve ETV6 (12p13.2) and reports suggest that native ETV6 deletions in ETV6RUNX1+ childhood ALL are associated with a favorable treatment outcome. Recent data indicates no difference in overall outcome between childhood ALL cases with vs. without $12 \mathrm{p}$ abnormalities (Heerema, 2000). In this study, we observed terminal deletions in $12 p$ at 3 different breakpoints (p11.2,p12 and p13) to co-exist with hyperdiploidy, hypodiploidy, $\operatorname{del}(21)(q 22), \operatorname{del}(6)(q 15)$, $+16,+21, \operatorname{del}(5)(\mathrm{q} 33 \mathrm{q} 35),+8$, and $\mathrm{i}(9)(\mathrm{q} 12)$.

Chromosomal rearrangements of MLL (11q23) gene are associated with high-risk acute leukemias (Pui et al., 2003). In the present study, del(11q23) occurred in cases with $\mathrm{t}(1 ; 19)(\mathrm{q} 23 ; \mathrm{p} 13)$, hypodiploidy and XYY karyotype. In ALL, del $(6 q)$ occurs as a sole anomaly (30\% of cases) or in association with specific abnormalities like del(12p), $\operatorname{del}(9 \mathrm{p}), \mathrm{t}(4 ; 11), \mathrm{t}(1 ; 19), \mathrm{t}(9 ; 22), \mathrm{t}(12 ; 21)$ or with random 
chromosomal changes (Brigaudeau $\mathrm{C}$ and Bilhou-Nabera C, 1998). In the present study, we observed terminal deletion at 6q15 along with $\operatorname{del}(12)(\mathrm{p} 11.2)$; and interstitial deletion at $6 \mathrm{q} 13 \mathrm{q} 21$ as part of a complex karyotype.

Deletion of chromosome arm 3p25/p26 and 9p/q is frequent in hematologic malignancies (Johansson et al., 1997). Furthermore, 9p abnormalities may hold significant negative impact on survival in adult BCP-ALL patients (Nahi et al., 2008). In this study, we observed deletion at 3p25-26 in a hypodiploid infant, in a complex karyotype and as a sole abnormality; while deletion at 9p/q occurred in a complex karyotype, with XXY karyotype and as a sole anomaly. Isochromosomes are relatively unusual and those readily identified in 1-4\% childhood ALL include $\mathrm{i}(6 \mathrm{p}), \mathrm{i}(7 \mathrm{q}), \mathrm{i}(9 \mathrm{q})$, and $\mathrm{i}(17 \mathrm{q})$. Both $\mathrm{i}(9 \mathrm{q})$ and $\mathrm{i}(7)$ occur in ALL either as a sole anomaly or in combination with non-random translocations like $\mathrm{t}(1 ; 19), \mathrm{t}(9 ; 22)$ and $(4 ; 11)$ (Pui et al., 1992).

In conclusion, this study reveals that chromosomal aberrations are frequent in de novo Indian B-lineage ALL patients. Furthermore their frequency and distribution differs between childhood and adult patients, and also differs between Indian and Western settings. Hence the potential impact of these differences on clinical behavior and treatment outcome merits further investigation. Our study bears important epidemiological data and confirms previous reports demonstrating the identity of unusual complex clonal chromosome aberrations in B-lineage ALL, suggesting that it is heterogeneous in its pathogenesis. Similarities and dissimilarities of the present findings with those of other researchers may be attributed to the influence of differential environmental exposure to unknown carcinogenic agents, differences in geographical distribution and ethnic origins.

\section{Acknowledgements}

The authors are grateful to the management of SRL Ltd for providing the necessary infrastructure facilities. No other research support is associated with this work.

\section{References}

Alonso CN, Gallego MS, Rossi JG, et al (2012). RT-PCR diagnosis of recurrent rearrangements in pediatric acute lymphoblastic leukemia in Argentina. Leuk Res, 36, 704-8.

Amare P, Gladstone B, Varghese C, Pai S, Advani S (1999). Clinical Significance of Cytogenetic Findings at Diagnosis and in Remission in Childhood and Adult Acute Lymphoblastic Leukemia: Experience from India. Cancer Genet Cytogenet, 110, 44-53.

Arora RS, Eden TOB, Kapoor G (2009). Epidemiology of childhood cancer in India. Indian J Cancer, 46, 264-73.

Awan T, Iqbal Z, Aleem A, et al (2012). Five most common prognostically important fusion oncogenes are detected in the majority of Pakistani pediatric acute lymphoblastic leukemia patients and are strongly associated with disease biology and treatment outcome. Asian Pac J Cancer Prev, 13, 5469-75.

Bhandari P, Ahmad F, Dalvi R, et al (2014). Molecular cytogenetic investigations in a novel chromosomal abnormality of $\mathrm{t}(10 ; 15)(\mathrm{q} 22 ; \mathrm{q} 22)$ in a pediatric precursorb-acute lymphoblastic leukemia patient. J Hematol Oncol
Res, 1, 28-33.

Bhatia P, Binota J, Varma N, et al (2012). Incidence of common chimeric fusion transcripts in B-cell acute lymphoblastic leukemia: an Indian perspective. Acta Haematol, 128, 17-9.

Brigaudeau C, Bilhou-Nabera C (1998). del(6q) abnormalities in lymphoid malignancies. Atlas Genet Cytogenet Oncol Haematol, 3, 33-5.

Campbell LJ, Martinow A, Michael PM, et al (1999). Correlation of cytogenetics, BCR-ABL PCR studies and fluorescence in situ hybridisation (FISH) in adult acute lymphoblastic leukaemia. Aust N Z J Med, 29, 707-12.

Ceppi F, Brown A, Betts DR, Niggli F, Popovic MB (2009). Cytogenetic characterization of childhood acute lymphoblastic leukemia in Nicaragua. Pediatr Blood Cancer, 53, 1238-41.

Chen B, Wang Y-Y, Shen Y, et al (2012). Newly diagnosed acute lymphoblastic leukemia in China (I): abnormal genetic patterns in 1346 childhood and adult cases and their comparison with the reports from Western countries. Leukemia, 26, 1608-16.

De Braekeleer E, Basinko A, Douet-Guilbert N, et al (2010). Cytogenetics in pre-B and B-cell acute lymphoblastic leukemia: a study of 208 patients diagnosed between 1981 and 2008. Cancer Genet Cytogenet, 200, 8-15.

Fauzdar A, Jain D, Mishra M, et al (2010). Molecular cytogenetic study in pediatric b-lineage acute lymphoblastic leukemia (BCP-ALL): A collaborative study group from North India. J Clin Oncol.

Gaynon PS, Trigg ME, Heerema NA, et al (2000). Children's Cancer Group trials in childhood acute lymphoblastic leukemia: 1983-1995. Leukemia, 14, 2223-33.

GFCH (1996). Cytogenetic abnormalities in adult acute lymphoblastic leukemia: correlations with hematologic findings outcome. A collaborative study of the group français de cytogenetique hematologique. Blood, 87, 3135-42.

Hann I, Vora A, Harrison G, et al (2001). Determinants of outcome after intensified therapy of childhood lymphoblastic leukaemia: results from medical research council united kingdom acute lymphoblastic leukaemia XI protocol. $\mathrm{Br} J$ Haematol, 113, 103-14.

Harrison CJ, Haas O, Harbott J, et al (2010). Detection of prognostically relevant genetic abnormalities in childhood B-cell precursor acute lymphoblastic leukaemia: recommendations from the biology and diagnosis committee of the international berlin-frankfurt-munster study group. $\mathrm{Br}$ J Haematol, 151, 132-42.

Heerema N (2000). 12p rearrangements in ALL. Atlas Genet Cytogenet Oncol Haematol, 4, 20-1.

Heerema NA, Nachman JB, Sather HN, et al (1999). Hypodiploidy with less than 45 chromosomes confers adverse risk in childhood acute lymphoblastic leukemia: a report from the children's cancer group. Blood, 94, 4036-45.

Hill A, Short MA, Varghese C, et al (2005).The $t(12: 21)$ is underrepresented in childhood B-lineage acute lymphoblastic leukemia in Kerala, Southern India. Haematologica, 90, 414-6.

Hunger SP, Lu X, Devidas M, et al (2012). Improved survival for children and adolescents with acute lymphoblastic leukemia between 1990 and 2005: a report from the children's oncology group. J Clin Oncol, 30, 1663-9.

Iacobucci I, Papayannidis C, Lonetti A, et al (2012). Cytogenetic and molecular predictors of outcome in acute lymphocytic leukemia: recent developments. Curr Hematol Malig Rep, 7, 133-43.

Jimenez-Morales S, Miranda-Peralta E, Saldana-Alvarez Y, et al (2008). BCR-ABL, ETV6-RUNX1 and E2A-PBX1: prevalence of the most common acute lymphoblastic 
leukemia fusion genes in Mexican patients. Leuk Res, 32, 1518-22.

Johansson B, Billstrom R, Kristoffersson U, et al (1997). Deletion of chromosome arm $3 p$ in hematologic malignancies. Leukemia, 11, 1207-13.

Mancini M, Scappaticci D, Cimino G, et al (2005). A comprehensive genetic classification of adult acute lymphoblastic leukemia (ALL): analysis of the GIMEMA 0496 protocol. Blood, 105, 3434-41.

Mazloumi SHM, Madhumathi DS, Appaji L, Prasannakumari (2012). Combined study of cytogenetics and fluorescence in situ hybridization (FISH) analysis in childhood acute lymphoblastic leukemia (ALL) in a tertiary cancer centre in South India. Asian Pac J Cancer Prev, 13, 3825-7.

Mesquita DR, Cordoba JC, Magalhaes IQ, et al (2009). Molecular and chromosomal mutations among children with B-lineage lymphoblastic leukemia in Brazil's Federal District. Genet Mol Res, 8, 345-53.

Moorman AV, Chilton L, Wilkinson J, et al (2010a). A population-based cytogenetic study of adults with acute lymphoblastic leukemia. Blood, 115, 206-14.

Moorman AV, Ensor HM, Richards SM, et al (2010). Prognostic effect of chromosomal abnormalities in childhood B-cell precursor acute lymphoblastic leukaemia: results from the UK medical research council ALL97/99 randomised trial. Lancet Oncol, 11, 429-38.

Nahi H, Hägglund H, Ahlgren T, et al (2008). An investigation into whether deletions in $9 \mathrm{p}$ reflect prognosis in adult precursor B-cell acute lymphoblastic leukemia: a multicenter study of 381 patients. Haematologica, 93, 1734-8.

Pallisgaard N, Hokland P, Riishoj DC, Pedersen B, Jorgensen P (1998). Multiplex reverse transcription-polymerase chain reaction for simultaneous screening of 29 translocations and chromosomal aberrations in acute leukemia. Blood, 92, 574-88.

Pandita A, Harish R, Digra SK, et al (2015). Molecular cytogenetics in childhood acute lymphoblastic leukemia: a hospital-based observational study. Clin Med Insights Oncol, 9, 39-42.

Polampalli S, Prabhash K, Amare P, et al (2011). Role of RT-PCR and FISH in diagnosis and monitoring of acute promyelocytic leukemia. Indian J Cancer, 48, 60 .

Pui C-H, Carroll A, Raimondi S, et al (1992). Isochromosomes in childhood acute lymphoblastic leukemia: A collaborative study of 83 cases. Blood, 79, 2384-91.

Pui C-H, Chessells JM, Camitta B, et al (2003). Clinical heterogeneity in childhood acute lymphoblastic leukemia with 11q23 rearrangements. Leukemia, 17, 700-6.

Pui C-H, Evans WE (2006). Treatment of acute lymphoblastic leukemia. $N$ Engl J Med, 354, 166-78.

Sabir N, Iqbal Z, Aleem A, et al (2012). Prognostically significant fusion oncogenes in Pakistani patients with adult acute lymphoblastic leukemia and their association with disease biology and outcome. Asian Pac J Cancer Prev, 13, 3349-55.

Safaei A, Shahryari J, Farzaneh MR, Tabibi N, Hosseini M (2013). Cytogenetic findings of patients with acute lymphoblastic leukemia in fars province. Iran $\mathrm{J} \mathrm{Med} \mathrm{Sci,}$ 38, 301-7.

Santamaria-Quesada C, Vargas M, Venegas P, et al (2009). Molecular and epidemiologic findings of childhood acute leukemia in Costa Rica. J Pediatr Hematol Oncol, 31, 131-5.

Sazawal S, Bhatia K, Gutierrez MI, et al (2004). Paucity of TELAML 1 translocation, by multiplex RT-PCR, in B-lineage acute lymphoblastic leukemia (ALL) in Indian patients. $A m$ J Hematol, 76, 80-2.

Secker-Walker LM, Prentice HG, Durrant J, et al (1997). Cytogenetics adds independent prognostic information in
DOI:http://dx.doi.org/10.7314/APJCP.2015.16.16.7219

Chromosomal Aberrations in Indian B Lineage ALL Patients adults with acute lymphoblastic leukaemia on MRC trial UKALL XA. MRC Adult Leukaemia Working Party. $\mathrm{Br} J$ Haematol, 96, 601-10.

Shaffer LG, McGowan-Jordan J, Schmid M, editors (2013). ISCN 2013: An International System for Human Cytogenetic Nomenclature. Karger, Basel.

Shaikh MS, Ali SS, Khurshid M, Fadoo Z (2014). Chromosomal abnormalities in Pakistani children with acute lymphoblastic leukemia. Asian Pac J Cancer Prev, 15, 3907-9.

Siraj AK, Kamat S, Gutierrez MI, et al (2003). Frequencies of the major subgroups of precursor B-cell acute lymphoblastic leukemia in Indian children differ from the West. Leukemia, 17, 1192-3.

Stark B, Jeison M, Gobuzov R, et al (2001). Near haploid childhood acute lymphoblastic leukemia masked by hyperdiploid line: detection by fluorescence in situ hybridization. Cancer Genet Cytogenet, 128, 108-13.

Walters R, Kantarjian HM, Keating MJ, et al (1990). The importance of cytogenetic studies in adult acute lymphocytic leukemia. Am J Med, 89, 579-87.

Watanabe N, Kobayashi H, Ichiji O, et al (2003). Cryptic insertion and translocation or nondividing leukemic cells disclosed by FISH analysis in infant acute leukemia with discrepant molecular and cytogenetic findings. Leukemia, 17, 876-82. 